\title{
Lung carcinoma recognition by blood dielectric spectroscopy
}

\author{
Xiaoyan Chen ${ }^{\mathrm{a}, *}$, Xinqiang $\mathrm{Lv}^{\mathrm{a}}$ and Huaxiang Wang ${ }^{\mathrm{b}}$ \\ ${ }^{a}$ Tianjin University of Science and Technology, 300222, Tianjin, China \\ ${ }^{b}$ Tianjin University, 300072, Tianjin, China
}

\begin{abstract}
Lung carcinoma has become one of the malignancies whose morbidity and mortality are growing significantly. Blood is a functional body fluid, it delivers oxygen and nutrients to the other parts of the body, and it is a pathway of tumor metastasis. This paper studies the possibility of diagnosing lung carcinoma by blood dielectric spectroscopy. Comparison experiments were carried out to trace the dielectric spectroscopy of blood with and without lung carcinoma changing at different tumor stages from $100 \mathrm{~Hz}$ to $100 \mathrm{MHz}$. The research results show that the discrepancy of complex permittivity between rat blood with and without lung carcinoma becomes significant with tumor growing, which is worthy for clinical diagnosis.
\end{abstract}

Keywords: Lung carcinoma, dielectric spectroscopy, blood, noninvasive detection

\section{Introduction}

Lung carcinoma has been seriously threatening human life in recent years. According to the relevant reports, the morbidity and mortality of lung carcinoma are higher than other malignant tumors [1]. The current clinical diagnostic methods for lung carcinoma includes X-ray slice, bronchoscope image, cytological examination, and enhanced CT slice [2,3]. However, the indistinguishable characteristics of the early tumor cells make it tough to detect early lung carcinoma by existing methods.

The dielectric spectroscopy measurement has received considerable attentions during the past two decades with the advantages including low cost, easy application, non-invasiveness and on-line monitoring [4]. As the electrical characteristics of cells are affected by the intracellular, extracellular and the activity of the membrane, the changes within and around the cell can be reflected in the dielectric spectroscopy. Y. Polevaya, et al. [5] verified the dielectric difference between normal and malignant white blood cells. Egot-Lemaire, et al. [6] compared normal human lung and malignant cells by dielectric spectroscopy in the range of $200 \mathrm{MHz}$ to $2 \mathrm{GHz}$. Although the research has made improvements in the diagnosis of lung carcinoma [7-10], few are related to the dielectric spectroscopy of a carcinoma through whole blood. This paper attempts to find an approach of diagnosing lung carcinoma by whole blood dielectric spectroscopy. Comparison experiments are carried out to trace the dielectric spectroscopy of whole blood with and without lung carcinoma. The diversity of the

\footnotetext{
* Address for correspondence: Xiaoyan Chen, Tianjin University of Science and Technology, 300222, Tianjin, China. Tel.: 13752113103; Fax: (86)22-60275497; E-mail: cxywxr@tust.edu.cn.
} 
spectroscopy at different frequencies and different stages are analyzed, expecting to discover the feasibility of using dielectric spectroscopy of whole blood to diagnose the carcinoma.

\section{Materials and methods}

\subsection{Materials and measurement system}

The dielectric spectroscopy was investigated in a prospective animal trial of 12 male rats (C57BL/6, weighing 18 22 g). The measurement system (Figure 1) consists of Agilent4294A (Agilent Technology, USA), adapter 16092A (Agilent Technology, USA), cylindrical measuring cell $(0.12 \mathrm{ml}$, produced by transparent PMMA (polymethyl methacrylate)), measuring electrodes (made of copper, which has high conductivity) and a laptop. Blood collections are implemented by vacuum blood collection tubes, disposable micro centrifuge tube, syringe, heparin, and physiological saline $(0.9 \%)$. It is reported that increasing of heparin content up to $0.35 \mathrm{~mL}$ per $10 \mathrm{~cm}^{3}$ does not change the results [11]. Therefore, measuring cell walls are coated with heparin less than that level in the experiments (approximately $0.15 \mathrm{~mL}$ per $10 \mathrm{~cm}^{3}$ ).

The rats were divided randomly into two groups: an experimental group with 8 rats, and a control group with 4 rats. Each rat in the experimental group was inoculated with $0.1 \mathrm{~mL}$ Lewis lung carcinoma cell suspension (The percentage of live carcinoma cells is over 95\%) outside the right forelimb axillaries. The two groups were raised in the same environment (temperature of $18 \sim 22^{\circ} \mathrm{C}$, humidity of $50 \% \sim 60 \%$ ).

\subsection{Measurement}

The rats with lung carcinoma were measured after 7 days after injection with Lewis lung carcinoma cell suspension. Every measurement is taken with 7 day intervals until the rats died. A volume of 0.2 $\mathrm{ml}$ bloods were collected from each rat respectively each time. Orbital blood and tail artery blood are collected bi-weekly to ensure the blood quantity and keep rats alive. Besides, measurement conditions and procedures were controlled strictly to ensure stability and repeatability of the measurements.

In order to avoid coagulation, blood was moved quickly into the measuring cell by the syringe (coated with heparin). The system was calibrated with open, short and scaled loads before

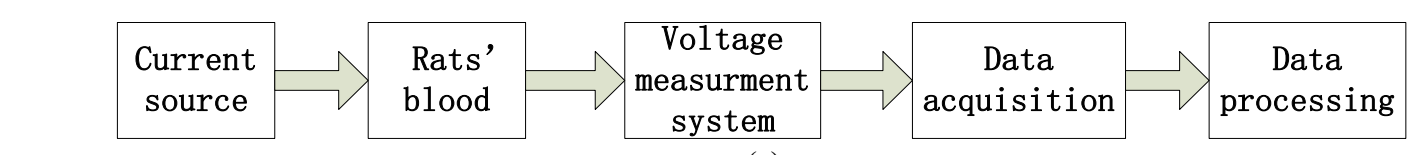

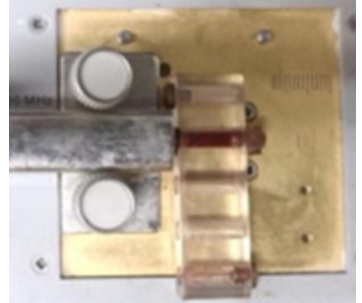

(b)

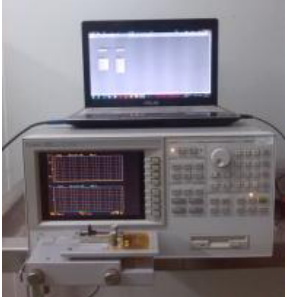

(c)

(a)

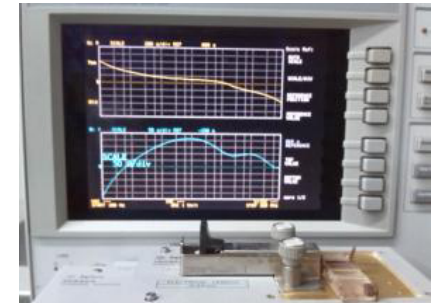

(d)

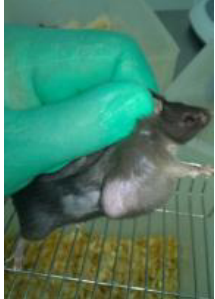

(e)

Fig. 1. Blood dielectric spectroscopy measurement system. (a) Measurement system diagram. (b) Filled measuring cell and adapter. (c) Laptop andAgilent4294A. (d) Display panel of Agilent4294A. (e) Lung carcinoma of a rat. 


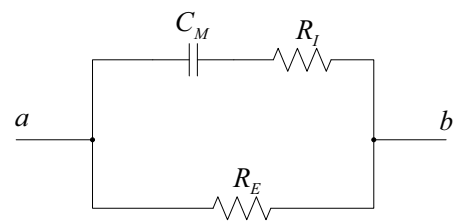

Fig. 2. The three elements equivalent circuit of a cell.

measurement. The measuring cell is filled with blood to guarantee the electrodes are covered and then connected with the adapter 16092A.The resistance (R) and inductance (X) at 31 points logarithmically distributed from $100 \mathrm{~Hz} 100 \mathrm{MHz}$ were measured under R-X model of Agilent4294A.

\subsection{Data processing}

According to the Cole-Cole theory [12], a cell can be expressed by three electrical elements (Figure 2), where $R_{E}$ refers to the equivalent resistance of extracellular fluid, $C_{M}$ stands for the equivalent capacitance of the membrane, and $R_{I}$ indicates the equivalent resistance of intracellular fluid. Equivalent impedance $Z$ between port $a$ and $b$ can be presented by Eq. (1), in which $R$ represents the real part of $Z$, indicating the resistance of the cell, and $X$ represents the imaginary part of $Z$, indicating the inductance of the cell.

The conductivity and permittivity of blood can be deduced through Eqs. (1)-(5).

$$
\begin{gathered}
Z=R+j X \\
G=\frac{R \quad}{R^{2}+X^{2}}, C=\frac{1}{2 \pi f} \cdot \frac{-X}{R^{2}+X^{2}}
\end{gathered}
$$

Where $\mathrm{C}$ is the equivalent capacitance and $\mathrm{G}$ is the equivalent conductance. $j$ is an operator expressed square root of -1 .f is the frequency. Complex permittivity $\varepsilon^{*}$ and complex conductivity $K^{*}$ are

$$
\begin{gathered}
\varepsilon^{*}=\varepsilon^{\prime}+i \varepsilon^{\prime \prime}, K^{*}=K^{\prime}+i K^{\prime \prime} \\
\varepsilon^{\prime}=C d / \varepsilon_{0} S, K^{\prime}=d G / S \\
\varepsilon^{\prime \prime}=\left(K^{\prime}-K_{l}^{\prime}\right) / \omega \varepsilon_{0}, K^{\prime \prime}=\left(\varepsilon^{\prime}-\varepsilon_{h}^{\prime}\right) \omega \varepsilon_{0}
\end{gathered}
$$

Where $d=14 \times 10^{-3} \mathrm{~m}$ and $S=12.56 \times 10^{-6} \mathrm{~m}^{2}$ are the length and the cross-sectional area of measuring cell, respectively. The real part of complex permittivity is $\varepsilon^{\prime}$ and the imaginary part of complex permittivity is $\varepsilon^{\prime \prime}$, whereas $\varepsilon_{h}^{\prime}$ is the maximum value of $\varepsilon^{\prime}$. Likewise, $K^{\prime}$ is the real part of complex conductivity, and $K^{\prime \prime}$ is the imaginary part of complex conductivity, $K_{l}^{\prime}$ is the minimum value of $K^{\prime}$. The angular frequency is $\omega$. The vacuum permittivity is $\varepsilon_{0}=8.85419 \times 10^{-12} \mathrm{~F} / \mathrm{m}$.

In order to select reliable data and discover the differences between the two groups, commercial statistical software, Statistical Package for the Social Sciences (SPSS), was used to perform the Kruskal-Wallis and T test. 
Table 1

The results of $\mathrm{T}$ test at different measuring time

\begin{tabular}{llllll}
\hline & $7^{\text {th }}$ day & $14^{\text {th }}$ day & $21^{\text {st }}$ day & $28^{\text {th }}$ day & $32^{\text {nd }}$ day \\
\hline $\mathrm{P}\left(\varepsilon^{\prime}\right)$ & 0.93 & 0.924 & 0.75 & 0.573 & 0.515 \\
\hline $\mathrm{P}\left(\varepsilon^{\prime \prime}\right)$ & 0.921 & 0.778 & 0.408 & 0.383 & 0.292 \\
\hline
\end{tabular}

\section{Results and discussion}

$\mathrm{P}$ values of the $\mathrm{T}$ test at 5 different measuring times are shown in Table 1. It's obvious that both $\mathrm{P}$ $\left(\varepsilon^{\prime}\right)$ and $\mathrm{P}\left(\varepsilon^{\prime \prime}\right)$ are gradually reduced over time. In other words, the discrepancies between the two groups become more remarkable.

Table 2 illustrates $f-\varepsilon^{\prime}$ and $f-\varepsilon^{\prime \prime}$ spectral contrast at different measuring times. The horizontal axis represents the excitation frequency and the vertical axis represents the real part of complex permittivity $\varepsilon^{\prime}$ and the imaginary part of complex permittivity $\varepsilon^{\prime \prime}$, respectively. As shown in Table 2, the experimental group $\varepsilon^{\prime}$ and $\varepsilon^{\prime \prime}$ are higher than that of the control group every acquisition time. The imaginary part of complex permittivity of both groups decrease from $10^{7}$ to $10^{2}$ with the excitation frequency increasing, indicating that the dielectric properties of cell change from

Table 2

Spectral contrast of complex permittivity at different measuring time

$7^{\text {th }}$ day

$$
f-\varepsilon^{\prime}
$$

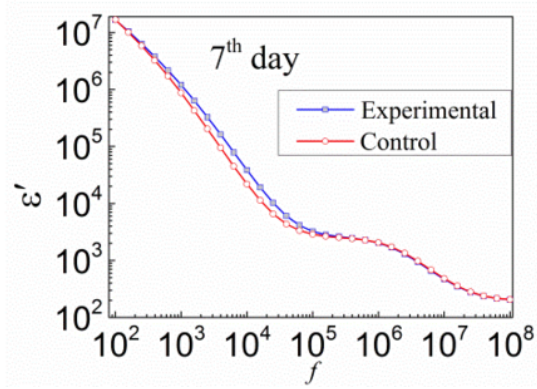

$14^{\text {th }}$

day

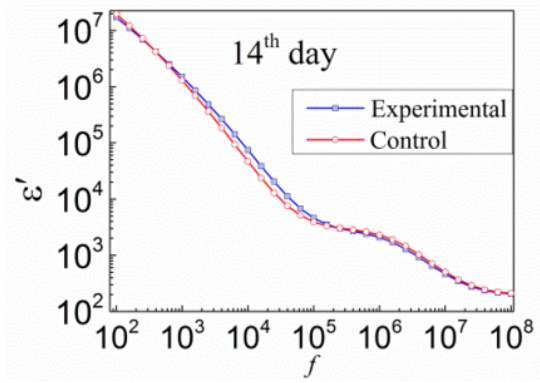

$f-\varepsilon$
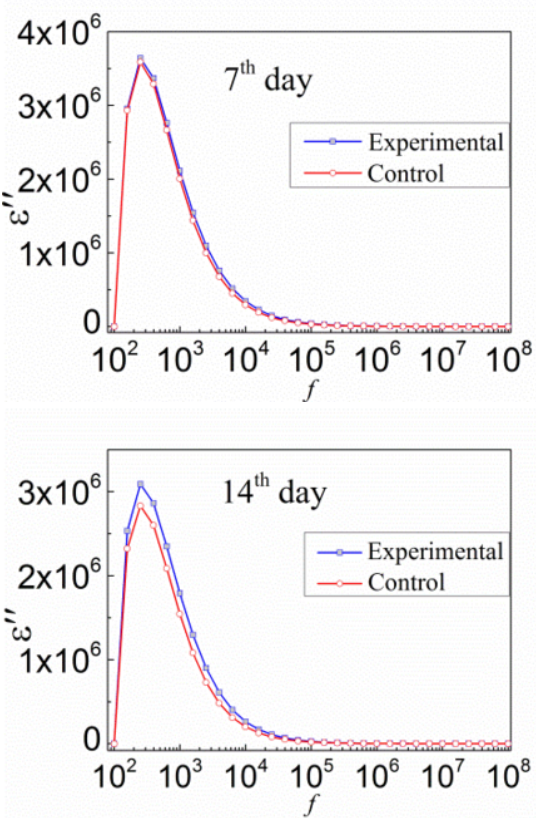
$21^{\text {st }}$

day

$28^{\text {th }}$

$32^{\text {nd }}$

day
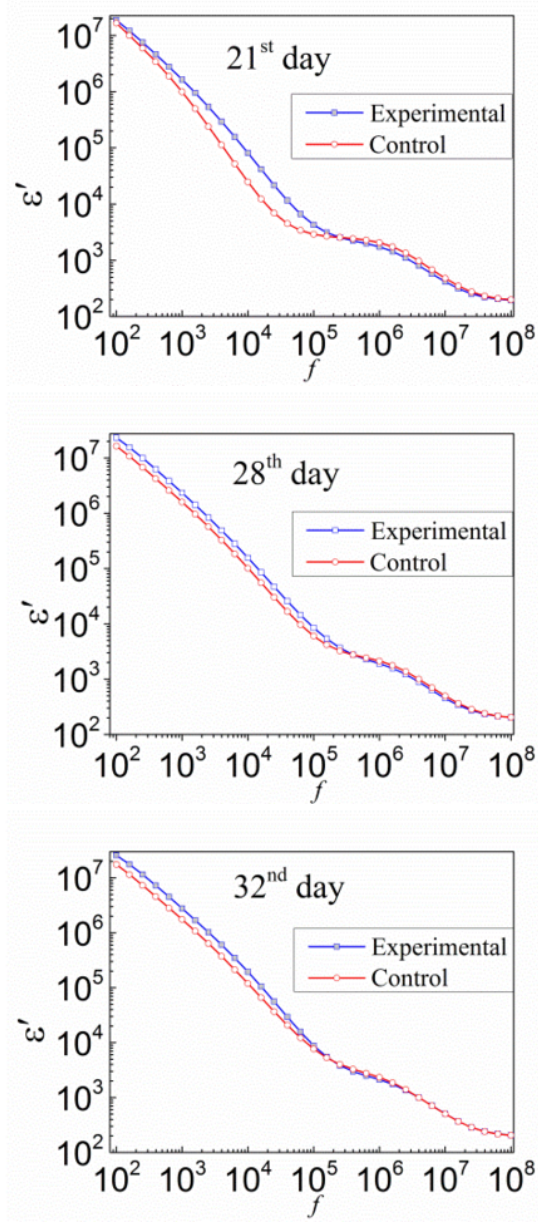
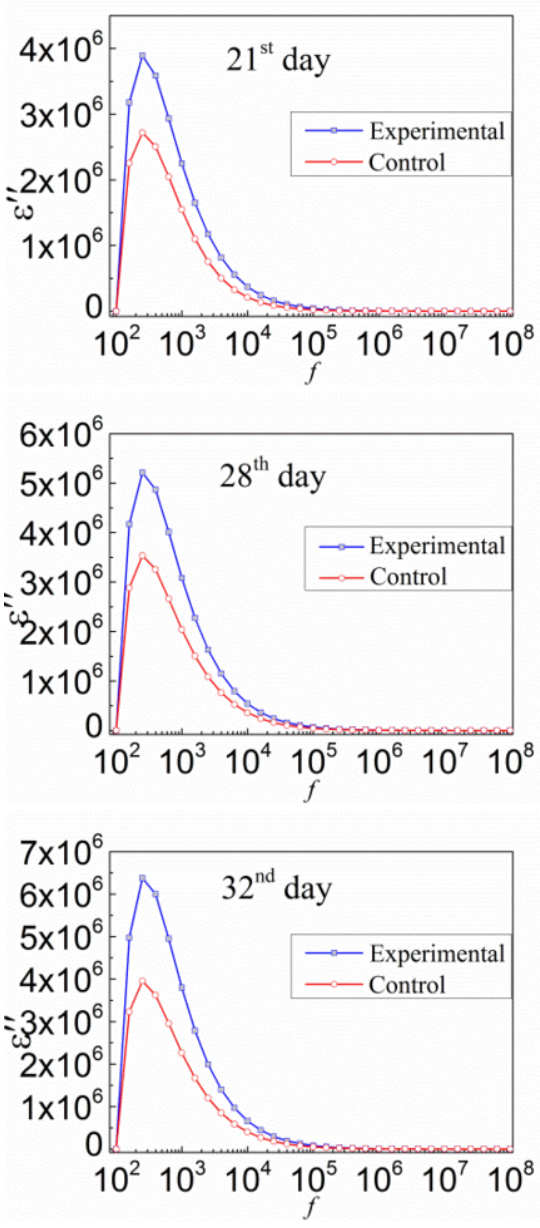

high resistance to low resistance. Besides, the spectral contrast results demonstrate that the distinctions between two groups become more and more obvious, which coordinates with the T test results.

Figure 3 presents the $f-\varepsilon$ 'spectral of two groups in different times. The horizontal axis represents the excitation frequency and the vertical axis represents the real part of the complex permittivity $\varepsilon$. The complex permittivity decreases gradually with frequency in both groups. The spectral shape shows the dispersion phenomenon from $10 \mathrm{KHz}$ to $100 \mathrm{KHz}$, which is consistent with Schwan's dispersion theory and indicates that the injected current goes through the cell membrane within this frequency area. It can be deduced that carcinoma cells have a negative effect on the dispersion phenomenon, for the dispersion phenomenon in spectral from later days are less obvious than that from earlier days (Figure 3(a)). Additionally, the most important trend in Figure 3(a) is that the value of $\varepsilon$ ' increase gradually with days, which doesn't appear in Figure 3(b).

The $f-\varepsilon "$ spectral of the two groups in different days are shown in Figure 4. The horizontal axis represents the excitation frequency and the vertical axis represents the imaginary part of complex 


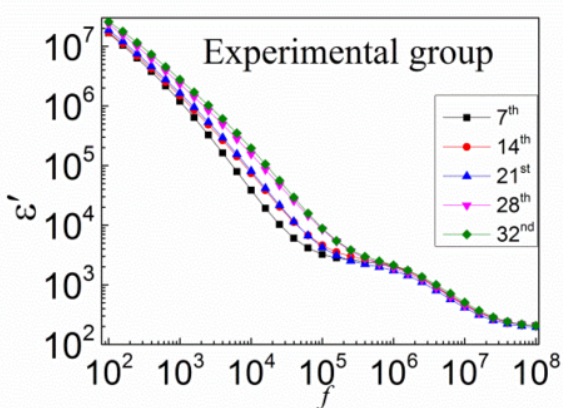

(a)

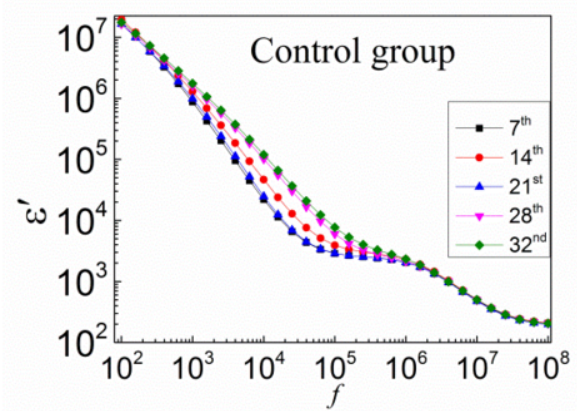

(b)

Fig. 3. The $f-\varepsilon^{\prime}$ Spectral of both two groups at different acquisition time (a) Experimental group. (b) Control group.

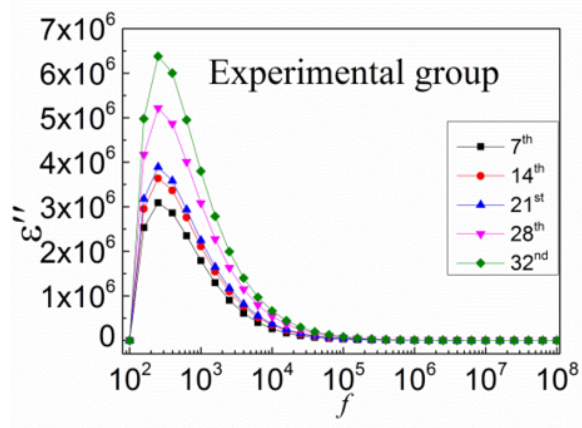

(a)

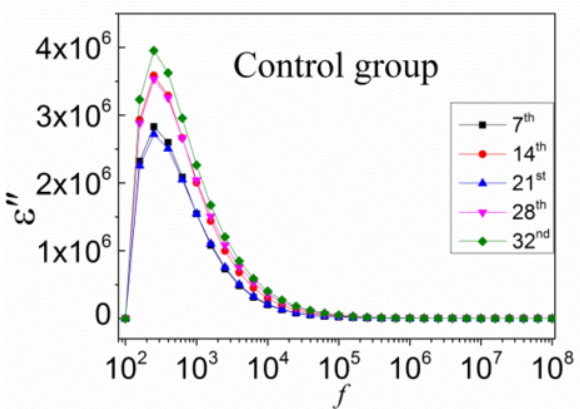

(b)

Fig. 4. The $f-\varepsilon "$ Spectral of both two groups at different acquisition time (a) Experimental group. (b)Control group.

permittivity $\varepsilon^{\prime \prime}$. The values of $\varepsilon^{\prime \prime}$ in both groups increase first and then reduce with frequency, and all the peaks appear around $500 \mathrm{~Hz}$. The most distinguishing part exists in the frequency range of 100 $\mathrm{Hz} \sim 100 \mathrm{KHz}$, and both $f-\varepsilon^{\prime}$ and $f-\varepsilon^{\prime \prime}$ curves show no distinguishing discrepancy over $100 \mathrm{KHz}$. It should be emphasized that in Figure 4(a) the peak of $\varepsilon$ " become higher and higher with time, which cannot be seen in Figure 4(b).Furthermore, the highest peak in experimental group (6379354.42) is 1.6 times higher than the one in the control group (3953617.83), and the amplitude of $\varepsilon$ " in experimental group always higher than the value in the control group.

As mentioned above, these results reveal the variations with times of the dielectric spectroscopy of blood with and without lung carcinoma. These remarkable regularities can be explained by the theory of tumor metastasis. Metastasis means the dissemination of carcinoma cells from the primary tumor to other organs, and it's the most frequent cause of death for patients with cancer [13, 14].The initial step in tumor metastasis is invasion of carcinoma cells into surrounding tissues and blood vessels by the extracellular matrix fibers. Once carcinoma cells reach blood vessels, then they must enter the blood space to travel to distant organs [15]. Thus, the difference of blood dielectric spectroscopy between normal and lung carcinoma rat is related to tumor metastasis. 


\section{Conclusion}

From above, two conclusions are drawn: 1) the difference between normal and lung carcinoma blood increases gradually with the measuring time, which means that the discrepancy becomes significant with tumor growing. Especially, the change of $\varepsilon "$ are stronger. 2) In the experimental group, the values of $\varepsilon$ ' and $\varepsilon^{\prime \prime}$ show a rising trend with days. The $f-\varepsilon$ " Spectral of blood can be considered as a fresh indicator in future diagnosis and treatment of lung carcinoma. The estimated of complex permittivity can be regarded as an electrical performance in clinical diagnosis of lung carcinoma. By tracing $\varepsilon$ " variation, it's convenient for doctors to achieve the patients' cancer information by a routine medical blood testing.

\section{Acknowledgment}

This work was funded by the National Natural Science Foundation of China (Grant No. 61301246, No. 61302122 and No. 61402330) and the Science and Technology Project of Tianjin (12JCYBJC19300). The authors wish to express special gratitude to Dr. Zhigang Qu and Dr. Juanita van Heerden for their valuable suggestions.

\section{References}

[1] H.J. Haussmann, Smoking and lung cancer: Future research directions, International Journal of Toxicology 26 (2007), 353-364.

[2] N.D. Magee, J.S. Villaumie, E.T. Marple, et al., Ex vivo diagnosis of lung cancer using a Raman miniprobe, Journal of Physical Chemistry 113 (2009), 8137-8141.

[3] F.R. Hirsch, W.A. Franklin, A.F. Gazdar, et al., Early detection of lung cancer: Clinical perspectives of recent advances in biology and radiology, Clinical Cancer Research 7 (2001), 5-22.

[4] X.Z. Dong, The development of the bioelectric impedance technologies, Chinese Journal of Medical Physics 21 (2004), 311-320.

[5] Y. Polevaya, Y. Feldman, B.Z. Ginzburg, et al., Study of normal and malignant white blood cells by time domain dielectric spectroscopy, IEEE Transactions on Dielectric and Electrical Insulation 82 (2001), 53-61.

[6] S. Egot-Lemaire, J. Pijanka, J. Sulé-Suso, et al., Dielectric spectroscopy of normal and malignant human lung cells at ultra-high frequencies, Physics in Medicine and Biology 54 (2009), 2341-2357.

[7] X.Y. Chen, X.Q Lv and M. Du, Experimental verification of depolarization effects in bioelectrical impedance measurement, Bio-Medical Materials and Engineering 24 (2014), 3675-3683.

[8] M .Wolf, R. Gulich, P. Lunkenheimer, et al., Broad band dielectric spectroscopy on human blood, Biochimica et Biophysica Acta 1810 (2011), 727-740.

[9] T. Dai and A. Adler, In vivo blood characterization from bioimpedance spectroscopy of blood pooling, IEEE Transaction on Instrumentation and Measurement 58 (2009), 3831-3837.

[10] C. Balan, C. Balut, L. Gheorghe, et al., Experimental determination of blood permittivity and conductivity in simple shear flow, Clinical Hemorheology and Microcirculation 30 (2004), 359-364.

[11] T. Chelidze, Dielectric spectroscopy of blood, Journal of Non-Crystalline Solids 305 (2002), 285-294.

[12] K.S. Cole and R.H. Cole, Dispersion and absorption in dielectrics I. Alternating current characteristics, The Journal of Chemical Physics 9 (1941), 341-351.

[13] H. Yamaguchi, J. Wyckoff and J. Condeelis, Cell migration in tumors, Current Opinion in Cell Biology 17 (2005), 559-564.

[14] A.F. Chambers, A.C. Groom and I.C. MacDonald, Dissemination and growth of cancer cells in metastatic sites, Nature Reviews Cancer 2 (2002), 563-572.

[15] F. van Zijl, G. Krupitza and W.G. Mikulits, Initial steps of metastasis: Cell invasion and endothelial transmigration, Mutation Research 728 (2011), 23-34. 